\title{
Smart plastic antibody material (SPAM) tailored on disposable screen printed electrodes for protein recognition: Application to myoglobin detection
}

\author{
Felismina T.C. Moreira, Sanjiv Sharma, Rosa A.F. Dutra, Joao P.C. Noronha, \\ Anthony E.G. Cass, M. Goreti F. Sales
}

\begin{abstract}
This work introduces two major changes to the conventional protocol for designing plastic antibodies:

(i) the imprinted sites were created with charged monomers while the surrounding environment was tailored using neutral material; and (ii) the protein was removed from its imprinted site by means of a protease, aiming at preserving the polymeric network of the plastic antibody. To our knowledge, these approaches were never presented before and the resulting material was named here as smart plastic antibody material(SPAM).

As proof of concept, SPAM was tailored on top of disposable gold-screen printed electrodes (Au-SPE), following a bottom-up approach, for targeting myoglobin (Myo) in a point-of-care context. The existence of imprinted sites was checked by comparing a SPAM modified surface to a negative control, consisting of similar material where the template was omitted from the procedure and called non-imprinted materials (NIMs). All stages of the creation of the SPAM and NIM on the Au layer were followed by both electrochemical impedance spectroscopy (EIS) and cyclic voltammetry (CV). AFM imaging was also performed to characterize the topography of the surface.

There are two major reasons supporting the fact that plastic antibodies were effectively designed by the above approach: (i) they were visualized for the first time by AFM, being present only in the SPAM network; and (ii) only the SPAM material was able to rebind to the target protein and produce a linear electrical response against EIS and square wave voltammetry (SWV) assays, with NIMs showing a similar-to-random behavior. The SPAM/AuSPE devices displayed linear responses to Myo in EIS and SWV assays down to $3.5 \mathrm{mg} / \mathrm{mL}$ and $0.58 \mathrm{mg} / \mathrm{mL}$, respectively, with detection limits of 1.5 and $0.28 \mathrm{mg} / \mathrm{mL}$.

SPAM materials also showed negligible interference from troponin T (TnT), bovine serum albumin (BSA) and urea under SWV assays, showing promising results for point-of-care applications when applied to spiked biological fluids.
\end{abstract}

Keywords

Plastic antibody, Charged binding site, Surface-molecular imprint, Screen-printed electrodes, Myoglobin, Biosensor

\section{Introduction}

Antibody-based immunoassays are the most commonly used diagnostic tool for the analysis of biomolecules (Borrebaeck, 2000; Katz and Davis, 2000; Reddy et al., 2011; Rimmer, 1998). This is due to the great selectivity and affinity linked to the antibody-antigen reactions observed in nature. Antibody-based assays however show several limitations, such as high production costs and low chemical stability (Ruigrok et al., 2011). In the presence of organic solvents, or in other non-physiological conditions such as high temperature, $\mathrm{pH}$ and high salt concentration, they lose their functionality.

An alternative approach to natural antibodies in immunoassays is to substitute them with synthetic materials displaying similar properties (Busi et al., 2004). Molecularly imprinted polymers, often referred to as plastic antibodies, are prepared by molecular imprinting technology, where a polymer is grown around the target molecule. Removal of the target from the polymerized matrix creates imprinted sites that match the size and shape of 
the target. Effective imprinted sites are expected to act similarly to natural antibodies, rebinding to the target compound with great affinity and selectivity.

Much has been achieved with successful imprinting of small size molecules (Chen et al., 2011), but the imprinting of proteins is still a challenge (Takeuchi and Hishiya, 2008; Turner et al., 2006; Whitcombe et al., 2011). Proteins by virtue of their complex structure have many potential recognition sites at their surface, such as charged amino acids and hydrophobic/hydrophilic regions. This makes the creation of molecular imprinted polymers with high selectivity challenging, due to possible cross-reactivity with proteins with similar charge or hydrophobic/hydrophilic structure as the template protein. Overall, the main constraints when imprinting proteins include their size, complexity, conformational flexibility and solubility (Turner et al., 2006). Different methods have been described so far for protein imprinting

(Takeuchi and Hishiya, 2008; Turner et al., 2006): (i) bulk imprinting, in which the whole protein is imprinted (Katz and Davis, 2000; Savina et al., 2009; Ye and Mosbach, 2001); (ii) epitope imprinting, where only the antigenic determinant part of the protein is used for imprinting (Bossi et al., 2012; LassNapiorkowska et al., 2012; Lu et al., 2012) and (iii) surface imprinting in which only a part of the surface of the protein is imprinted over a planar surface (Moreira et al. 2011a; Moreira et al., 2011b; Wang et al., 2008 Yildirim et al., 2012; Liao et al., 2009).

Despite its simplicity, bulk imprinting of proteins is today recognized as an approach of limited success. It is often associated with (i) irregular shape of the imprinted particles, (ii) poor template removal by protein entrapment and (iii) restricted stereochemical access of the protein to the rebinding sites (Whitcombe et al., 2011; Wang et al., 2008). Epitope imprinting seems to be a successful strategy (Whitcombe et al., 2011; Hillberg and Tabrizian, 2008), but only small to medium sized peptides have been imprinted so far and further studies are required to obtain unique and freely available epitope sequences (Hillberg and Tabrizian, 2008; Whitcombe et al., 2011).

When an entire protein is to be imprinted, surface imprinting is by far the most successful approach. It leads to higher binding capacity and faster mass transfer/bindingkinetics than traditional bulk processes. In general, surface imprinting takes place in 4 steps: (i) attachment of the template to the surface; (ii) selection of functional monomers showing a special ability to interact with the functional groups of the template molecule; (iii) a prepolymerization stage where these monomers are allowed to bind to the template molecule, followed by a polymerization step; and (iv) template removal, leaving behind cavities that constitute the templates comprising of empty rebinding sites.

Several strategies for surface imprinting have been described in the literature. Most of these report the use of immobilized template, initiation on the supporting matrix, and combined surface imprinting with controlled/living free radical polymerization (Chen et al., 2011; Shi et al., 1999; Bossi et al., 2007; Shiomi et al., 2005; Moreira et al., 2011a; Moreira et al., 2011b; Bonini et al., 2007; Piletsky et al., 2000). In general, the covalent immobilization of the template molecules at the surface of solid substrates offers some advantages: it enables imprinting templates that are insoluble in the pre-polymerization mixture and minimizes the template aggregation, thus leading to more homogeneous binding sites (Chen et al., 2011).

The monomers selected for creating the surface imprinted binding sites are at the core of the success of the plastic antibody operation (Andersson, 2000; Kriz et al., 1997; Shi et al., 1999). Due to the multiple-charge nature of proteins and their multiple surface features, different monomers have been employed, neutral or negatively/positively charged. In essence, they are expected to interact strongly with several points at the outer surface of the protein, thus favouring more specific template rebinding. However, as far as we are aware, the area surrounding the binding site has always been composed of the same material as the binding site, itself, which has contributed to non-specific interactions.

In order to create smart plastic antibody materials (SPAM) of enhanced specific binding, this work introduces for the first time charged monomers on the binding sites combined with neutral monomers in the rest of the polymeric matrix. This concept formed the basis for the design of Myo plastic antibodies over the gold surface of commercial screen-printed electrodes (AuSPE). Myo is the first cardiac biomarker found in the blood as an indicator of cardiac damage, which makes its detection in the first hours highly significant. The detection of myo should be made in point-of-care, quickly, and with low cost. The most advanced and commercially available devices so far (Alere Triage Ž Cardiac) include (always) immunoassay-based reactions.

The charged monomers used for this purpose were 2-acrylamido-2-methyl-1-propanesulfonic acid sodium salt (AMPSA) and 2-aminoethyl methacrylate hydrochloride (AEMA). The neutralsurrounding material was obtained by co-polymerizing acrylamide (AAm) and $\mathrm{N}, \mathrm{N}^{0}$-methylenebisacrylamide (NNMBA). Non-imprinted materials (NIM) were produced by removing the template from the procedure and the ability of the polymer to interact non-specifically with the template was measured. The resulting biosensor was evaluated by several electrochemical techniques and further applied to the analysis of biological samples.

\section{Experimental section}

\subsection{Apparatus}

The electrochemical measurements were conducted with a potentiostat/galvanostat from Metrohm Autolab and a PGSTAT302N, impedimetic module and controlled by Ivium software. Au-SPEs were purchased from DROPSENS (DRP-C220AT), having working and counter electrodes made of gold and reference electrode and electrical contacts made of silver. The diameter of the working electrode was $4 \mathrm{~mm}$ and the Au-SPEs were placed in a switch box (DROPSENS), interfacing the electrical contacts of the Au-SPE with the electrical connections of the potentiostat/galvanostat.

\subsection{Reagents}

All chemicals were of analytical grade and water was de-ionized or ultrapure Milli-Q laboratory grade. Potassium hexacyanoferrate III $\left(\mathrm{K}_{3}\left[\mathrm{Fe}(\mathrm{CN})_{6}\right]\right)$ and potassium hexacyanoferrate II $\left(\mathrm{K}_{4}\left[\mathrm{Fe}(\mathrm{CN})_{6}\right]\right)$ trihydrate were obtained from Riedel-deHaen; AAm, NNMBA, TnT, BSA, urea, and sodium chloride from Fluka; AMPSA, thiomalic acid (TMA) and AEMA from Aldrich; and Myo, N-hydroxysuccinimide (NHS), N-ethyl-No-(3-dimethylaminopropyl) carbodiimide hydrochloride (EDAC), and Benzoyl Peroxide (BPO) from Himedia.

\subsection{Solutions}

Stock solutions of $5.0 \times 10^{-6} \mathrm{~mol} / \mathrm{L}$ Myo were prepared in MES buffer $\left(1.0 \times 10^{-2} \mathrm{~mol} / \mathrm{L}, \mathrm{pH} 4.5\right)$. Less concentrated standards were obtained by accurate dilution of the previous solution in the same buffer. Electrochemical assays were performed with $5.0 \times 10^{-3} \mathrm{~mol} / \mathrm{L}$ $\mathrm{K}_{3}\left[\mathrm{Fe}(\mathrm{CN})_{6}\right]$ and $\mathrm{K}_{4}\left[\mathrm{Fe}(\mathrm{CN})_{6}\right]$ in MES $1.0 \times 10^{-3} \mathrm{~mol} / \mathrm{L}$, pH 4.5 . The selectivity study used $2.0 \times 10^{-7} \mathrm{~mol} / \mathrm{L}$ Myo solutions prepared in buffer and solutions of possible interfering species, TnT $(2.0 \mathrm{x}$ $\left.10^{-7} \mathrm{~mol} / \mathrm{L}\right)$, BSA $\left(2.0 \times 10^{-7} \mathrm{~mol} / \mathrm{L}\right)$, and urea $\left(2.0 \times 10^{-7} \mathrm{~mol} / \mathrm{L}\right)$, prepared in the same buffer. 


\subsection{Design of the plastic antibody on the Au-SPE}

The gold surface of the working electrode was thoroughly washed with de-ionized water and incubated in $10 \mathrm{mmol} / \mathrm{L}$ solutions of TMA for $20 \mathrm{~h}$, at $25 \mathrm{lC}$, previously. After this stage, all modifying solutions were applied subsequently and only on the working electrode area, by placing on it a $5 \mathrm{~mL}$ drop of the corresponding solution.

The carboxylic acid groups on the gold surface (Au-SPE/TMA) were activated by reaction with EDAC $50 \mathrm{mmol} / \mathrm{L}$ and $25 \mathrm{mmol} / \mathrm{L}$ NHS, and washed after with deionized water. The modified electrode was then incubated in Myo solution $1 \times 10^{-4} \mathrm{~mol} / \mathrm{L}$, prepared in PBS buffer, $\mathrm{pH} 7.0$, for $2.5 \mathrm{~h}$, at $4 \mathrm{lC}$. The SPE modified with Au/TMA/Myo was then thoroughly washed again with PBS buffer and ultrapure water to remove adsorbed Myo.

The imprinting stage started by incubating overnight the AuSPE/TMA/Myo electrode in $5.0 \times 10^{-4} \mathrm{~mol} / \mathrm{L}$ AMPSA and $5.0 \times 10^{-4} \mathrm{~mol} / \mathrm{L}$ AEMA. This was followed by the addition of $5.0 \times 10^{-4} \mathrm{~mol} / \mathrm{L}$ AAm (monomer), $5.0 \times 10^{-5} \mathrm{~mol} / \mathrm{L}$ NNMBA (cross-linker) and $5.0 \times 10^{-4} \mathrm{~mol} / \mathrm{L} \mathrm{BPO}$ (initiator) solutions. The polymerization was carried out at $25 \mathrm{lC}$ for $5 \mathrm{~h}$. The resulting material, Au-SPE/TMA/Myo/polymer, was thoroughly washed with deionized water and incubated overnight in protease $\mathrm{K}$, $400 \mathrm{mg} / \mathrm{mL}$, prepared in PBS buffer, $\mathrm{pH} 7.4$, in the dark, to remove the protein template. The resulting material without template, Au-SPE/TMA/SPAM, was then washed several times in PBS buffer to remove protein fragments and proteinase $\mathrm{K}$, and finally rinsed with MQ water (Zayats et al., 2011).

Blank materials of SPAM were produced in parallel, where the protein was removed from the synthetic pathway. These were named NIM (non-imprinted materials) and were used to evaluate the imprinting effect, because no specific binding sites should exist on it.

\subsection{AFM analysis}

The SPAM/NIM surfaces were characterized using atomic force microscopy. Two different modes of AFM were tried to characterize the surface of the electrodes. These were tapping mode (Veeco Metrology Multimode/Nanoscope IVA) and contact mode (Nanoink NScriptor based AFM with A type tips). AFM images from tapping mode were found better and Image $\mathrm{J}$ software was used to analyze these.

\subsection{Electrochemical procedures}

$\mathrm{CV}$ and SWV measurements were conducted in $5.0 \mathrm{mmol} / \mathrm{L}$ of $\left[\mathrm{Fe}(\mathrm{CN})_{6}\right]^{3-}$ and $5.0 \mathrm{mmol} / \mathrm{L}$ of $\left[\mathrm{Fe}(\mathrm{CN})_{6}\right]^{4-}$, prepared in MES buffer, $\mathrm{pH}$ 4.5. For CV assays, the potential was scanned from -0.6 to $\mathbf{p} 0.7 \mathrm{~V}$, at $50 \mathrm{mV} / \mathrm{s}$. In SWV studies potentials were changed from -0.6 to $\mathrm{p} 0.6 \mathrm{~V}$, at a frequency of $50 \mathrm{~Hz}$ with a step height of $150 \mathrm{mV}$. All assays were conducted in triplicate.

EIS assays were also conducted in triplicate with the same redox couple $\left[\mathrm{Fe}(\mathrm{CN})_{6}\right]^{3-/ 4-}$ at a standard potential of $\mathrm{p0} .12 \mathrm{~V}$, using a sinusoidal potential perturbation with an amplitude of $0.01 \mathrm{~V}$ and the number of frequencies equal to 50 , logarithmically distributed over a frequency range of $0.1-100 \mathrm{kHz}$. The impedance data were fitted to a Randles equivalent circuit using the implemented Ivium software.

Calibration curves were determined for Myo concentrations between 0.0250 and $41.1 \mathrm{mg} / \mathrm{mL}$. The time given for SPAM/Myo interaction was set to $5 \mathrm{~min}$. The response of the sensor was reversed after cleaning with proteinase $\mathrm{K}$ for $12 \mathrm{~h}$ and subsequent washing with PBS and water.

Selectivity studies were conducted by competitive assay between Myo, with a $2.0 \times 10^{-7} \mathrm{~mol} / \mathrm{L}$ concentration, and TnT,
BSA, or urea, solutions of the same concentration. All these were prepared in MES buffer $\mathrm{pH} 4.5$.

The standard addition method was applied to determine Myo in spiked serum samples with a BSA concentration. Myo concentration was set to $45 \mathrm{mg} / \mathrm{mL}$. This solution was further diluted to achieve Myo levels from 0.59 to $18.77 \mathrm{mg} / \mathrm{mL}$, lying within the linear range of the device.

\subsection{Binding isotherm}

The rebinding properties of Au-SPE/TMA/SPAM biosensorwere measured by calculating the apparent dissociation constant $\left(K_{\mathrm{D}}\right)$ through SWV assays. $K_{\mathrm{D}}$ was the protein concentration required to give half of the maximum response produced by the device. This was done by fitting to the Langmuir isotherm (Eq. (1)) where IS was the normalized current-density (in $\mathrm{Acm}^{-2}$ ), $S$, the concentration of Myo (in mol/L) and $I_{\max }$, the maximum current density observed (in $\mathrm{Acm}^{-2}$ ).

$I_{S}=\frac{I_{\max }}{1+K_{\mathrm{D}} /[\mathrm{S}]}$

\section{Results and discussion}

\subsection{SPAMdesign}

The preparation of SPAM involved a bottom-up approach of 4 stages, as indicated in Fig. 1. It started by assembling a carboxylic layer on top of the gold working electrode of the SPE. This layer was activated for covalently binding Myo. The imprinting stage started by positioning the charged-monomers on the pre-polymerization mixture and polymerizing the vacant sites around the protein with neutral monomer and cross-linker species. The imprinted sites were finally obtained once the protein was removed.

In detail, the carboxylic layer was formed first by incubating the Au-SPE in TMA (Fig. 1A). The contact of TMA with a clean gold substrate led to the spontaneous formation of a closely packed monolayer via a strong gold-sulfur interaction between the thiol group $(-\mathrm{SH})$ and $\mathrm{Au}$. The carboxylic groups pointing outside were then activated, aiming at binding the protein afterwards under near-to-physiological conditions. This was done by EDAC/NHS chemistry. This reaction is well known, forming a highly reactive O-Acylisourea intermediate that reacts quickly with NHS to produce a more stable succinimydyl ester intermediate (Jiang et al., 2004). This ester undergoes nucleophilic substitution with any readily available amine group (on Myo), resulting in the formation of an amide bond between the ester and protein (Fig. 1B).

The rebinding cavities expected to display a similar-toantibody behavior started being formed by self-organizing the charged-monomers around the protein. Each charged monomer would be bound by ionic interaction to a Myo residue of opposite charge located on the protein surface. By taking part in the subsequent polymerization stage, these monomers would remain in position within the final SPAM structure (Fig. 1C). The selection of the most suitable monomers for this purpose depended on the nature of the organic functions of Myo pointing towards the surrounding environment. Myo has many kinds of amino acid residues along its polypeptide chain of 153 amino acids, distributed over a globular and compact form ( $45 \times 35 \times 25 \AA)$. The exact amount and nature of the residues on the outer surface are however difficult to predict because proteins are not rigid objects and their biological function is controlled by conformational changes of different magnitudes (Brunori et al., 2004). So, one 


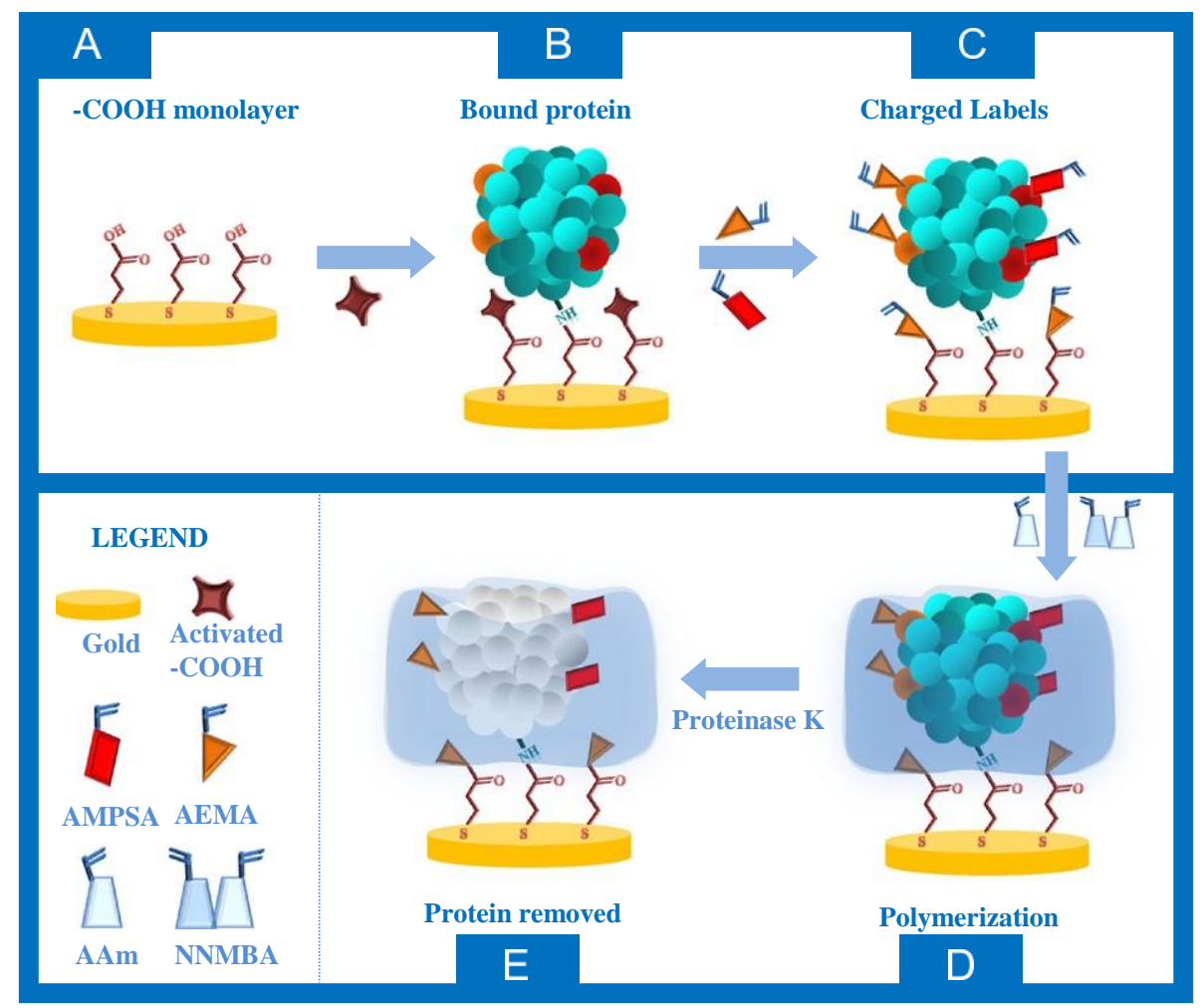

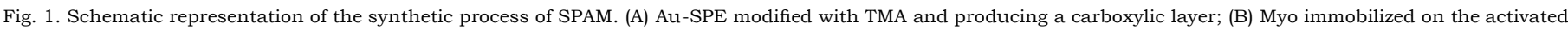

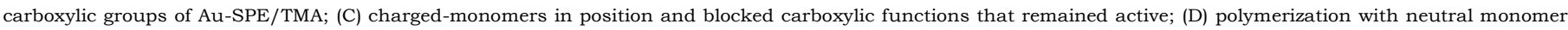
structures around the template; and $(\mathrm{E})$ : binding site formation by template removal with proteinase $\mathrm{K}$.

may say that both charged and uncharged polar amino acid sidechains are expected to be directed towards the external surface of the protein. Consequently, both positively and negatively charged-monomers were used, contributing to an increasing number of interactions, and thus to a more accurate stereochemical recognition of the protein template.

Monomers with amine (AEMA) and sulfonic acid (AMPSA) based functions were selected (Fig. 1C). Although they are not completely ionized in solution, they ensure physiological compatibility with the target protein, granting that its conformation is kept. Only the ionized fraction of these monomers interacting with Myo is expected to contribute to the imprinted site because weakly bound monomers were removed by washing the material with water after positioning the monomers. This was indeed an essential condition to ensure that only neutral monomers would take part in the subsequent polymerization stage (thus avoiding any polymerizable charged species outside the binding site area). In addition, the non-ionized fraction of the amine function also blocked the carboxylic functions remaining active after protein binding, thus avoiding side-reactions with the material and ensuring that the subsequent polymeric fraction would remain attached to the modified gold surface.

The position of the charged groups was set firm by polymerizing the vacant area around the protein (Fig. 1D). This was done by an addition reaction where all vinyl groups from monomer and cross-linker species gave rise to long branched carbon chains. Only amide functions were present in the final reticulated material because this was the only organic function in AAm and NNMBA monomers. A radical initiation of the polymerization was preferred (by using BPO) to cationic or anionic ones, because these would introduce a greater number of charged species inside the polymeric matrix. This matrix was firstly designed to be neutral outside the binding sites, and this was an essential condition to reduce non-specific-binding.
The SPAM material was ready after removing the template from its imprinted site (Fig. 1D). Many different approaches have been used for this purpose (Whitcombe et al., 2011; Zdyrko et al., 2009) and the use of a proteolytic enzyme is unique in this regard. This procedure was aimed at ensuring the complete removal of the protein and keeping mild conditions to avoid any disturbance around the SPAM network. Proteinase K was the enzyme selected for this purpose. It cleaved peptide bonds, converting each accessible single polypeptide from Myo into smaller peptide fragments. These fragments were removed from the cavity by rinsing the material with buffer.

\subsection{Control of the surface modification by impedance and voltammetry measurements}

The modification of metal surfaces with organic films produces global modifications in its electrical features. These can be measured by monitoring the changes in the electron transfer properties of well-known redox systems, such as $\left[\mathrm{Fe}(\mathrm{CN})_{6}\right]^{4-} /$ $\left[\mathrm{Fe}(\mathrm{CN})_{6}\right]^{3-}$. Indirect ways of measuring such alterations include EIS and CVassays.

EIS studies were used to follow the Au-SPE modification after each chemical change. Randle's equivalent circuit was adopted to model the physiochemical process occurring at the gold electrode surface as it is frequently used to interpret simple electrochemical systems. The elements of this circuit included the uncompensated resistance of the solution phase $\left(R_{\mathrm{S}}\right)$, the capacitance of the double layer $\left(C_{\mathrm{dl}}\right)$, and charge-transfer resistance $\left(R_{\mathrm{ct}}\right)$ which is inversely proportional to the rate of electron transfer, and the Warburg diffusion element $(W)$, accounting for the diffusion of ions from bulk electrolyte to the electrode interface.

The obtained EIS spectra are presented as Nyquist plots in Fig. 2. In general, the semicircle was observed at high frequency range and indicated a charge-transfer controlled process. The diameter of 


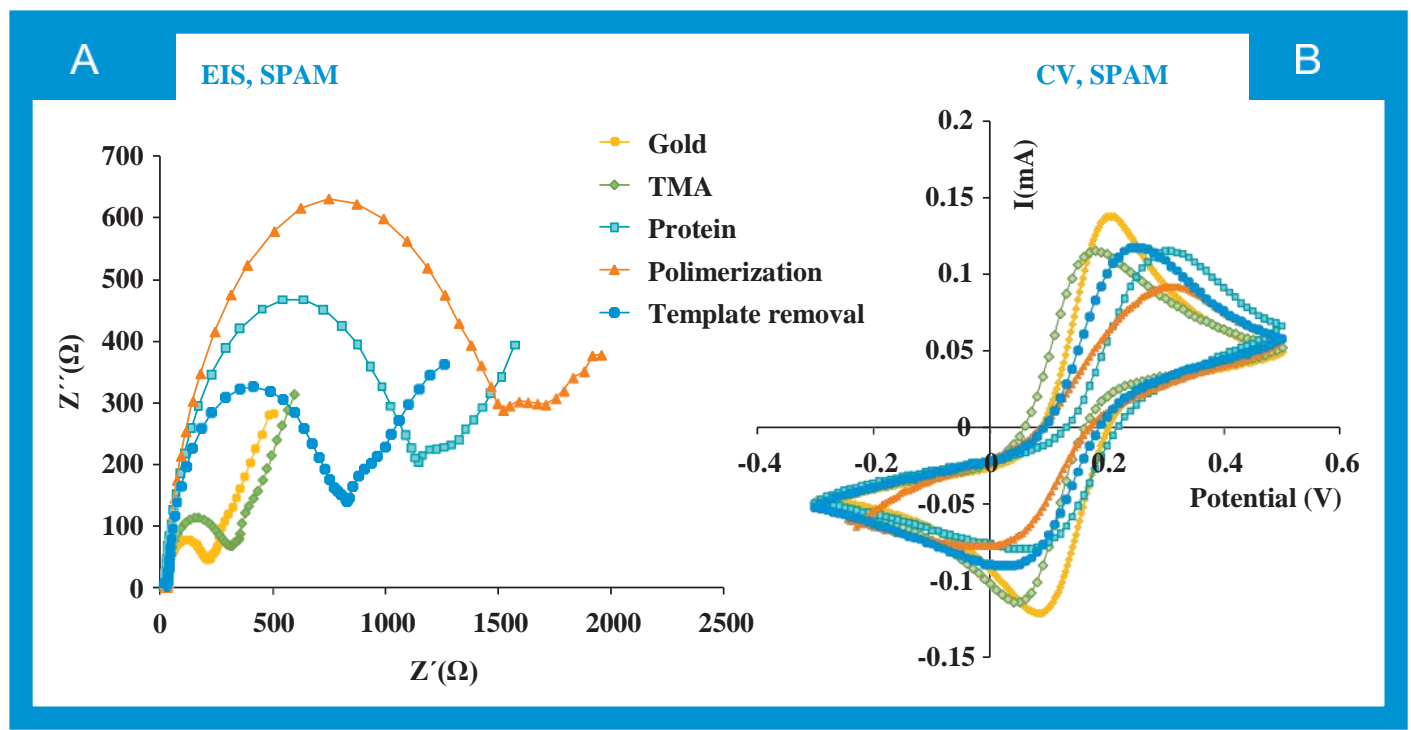

Fig. 2. Electrochemical study over the subsequent modification steps of the Au-SPE in $5.0 \mathrm{mM}\left[\mathrm{Fe}(\mathrm{CN})_{6}\right]^{3-}$ and $5.0 \mathrm{mM}\left[\mathrm{Fe}(\mathrm{CN})_{6}\right]^{4-}$, in $\mathrm{MES}$ buffer $\mathrm{pH} 4.5$, carried out by EIS (A, Nyquist plots) and CV (B, cyclic voltammograms) assays for SPAM.

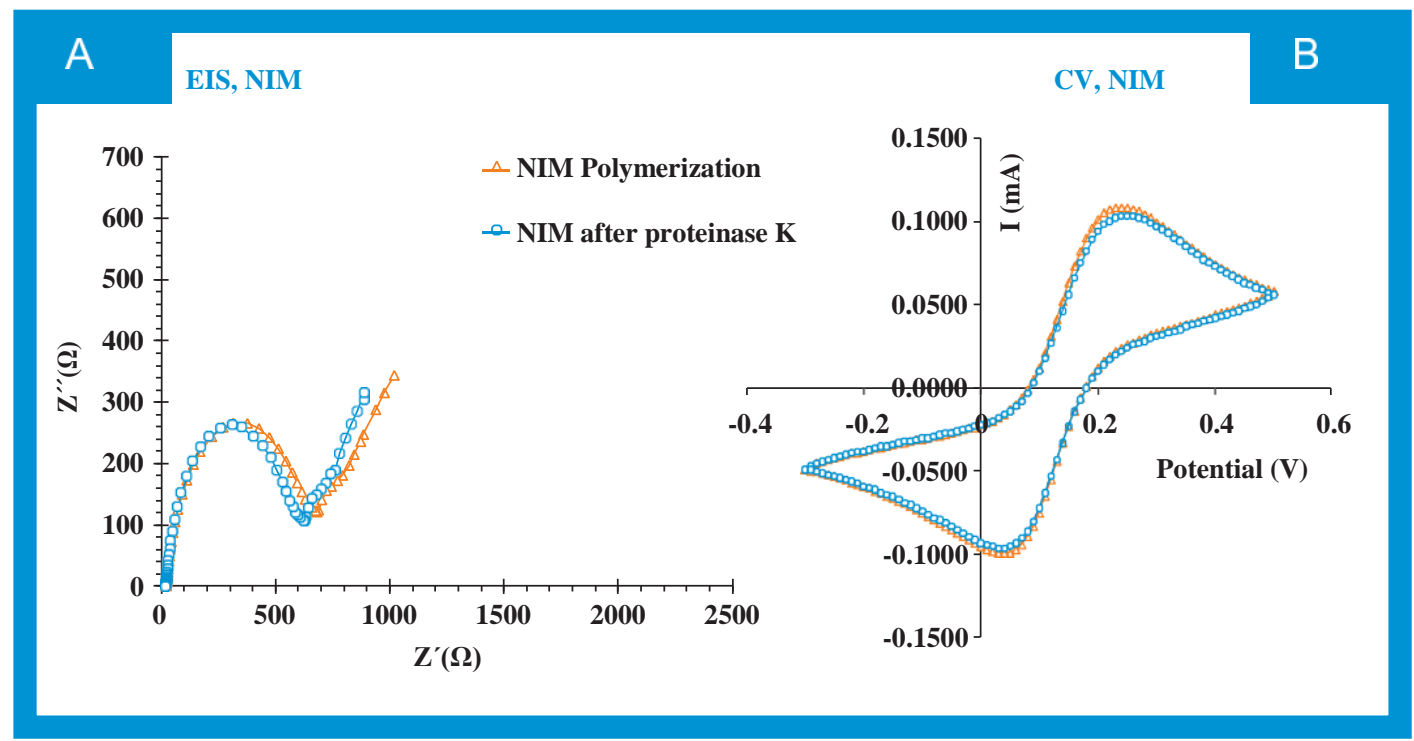

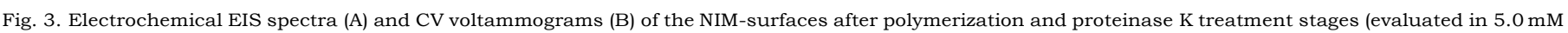
$\left[\mathrm{Fe}(\mathrm{CN})_{6}\right]^{3-}$ and $5.0 \mathrm{mM}\left[\mathrm{Fe}(\mathrm{CN})_{6}\right]^{4-}$, in MES buffer $\left.\mathrm{pH} 4.5\right)$.

this semicircle equaled $R_{\mathrm{ct}}$, controlling the electron transfer kinetics of the redox-probe at the electrode interface (Panagopoulou et al., 2010). The linear arm was seen in the low frequency range and showed diffusion-controlled mass-transfer.

The bare gold surface showed a very small semicircle domain, suggesting a very fast electron-transfer process with a diffusional limiting step (Fig. 2A). Modification with the TMA monolayer gave rise to subsequent increases in the electron transfer resistance, resulting in increases in the semicircular section of the Nyquist plot. For SPAM polymer (Fig. 2A), further linkage of Myo followed by the polymerization stage produced additional barriers for the redox probe access to the Au-SPE modified electrode. This resulted in an extra increase in the electron transfer resistance, reflected by further substantial increase in $R_{\text {ct }}$. The final step of the artificial antibody synthesis was the template removal with proteinase $\mathrm{K}$. In this step the resistance decreased (Fig. 2A), suggesting that Myo was successfully extracted from the polymer. No resistance decrease was observed on NIM after proteinase $\mathrm{K}$ treatment (Fig. 3A), which accounted for the stability of the polymer network against the enzyme. The $R_{\mathrm{ct}}$ of the NIM materials (Fig. 3A) was close to that of SPAM material without template (Figure S1-A), thus suggesting that the template removal step was efficient.

CV assays shown in Figs. 2B, 3B, and $\mathrm{S} 1-\mathrm{B}$ supported the previous studies of EIS. The modifications of the bare gold up to the polymerization stage increased the peak-to-peak potential separation and decreased the peak height of the redox probe in the voltammograms, thus accounting for the increased $R_{\text {ct. }}$. This behavior was reversed after the template removal, accounting for the absence of the protein and the polymer porosity.

\subsection{AFM analysis}

Although electrical characterization was done on SPEs, surface characterization studies were conducted only on planar gold surfaces employed in regular surface plasmon resonance (SPR) 


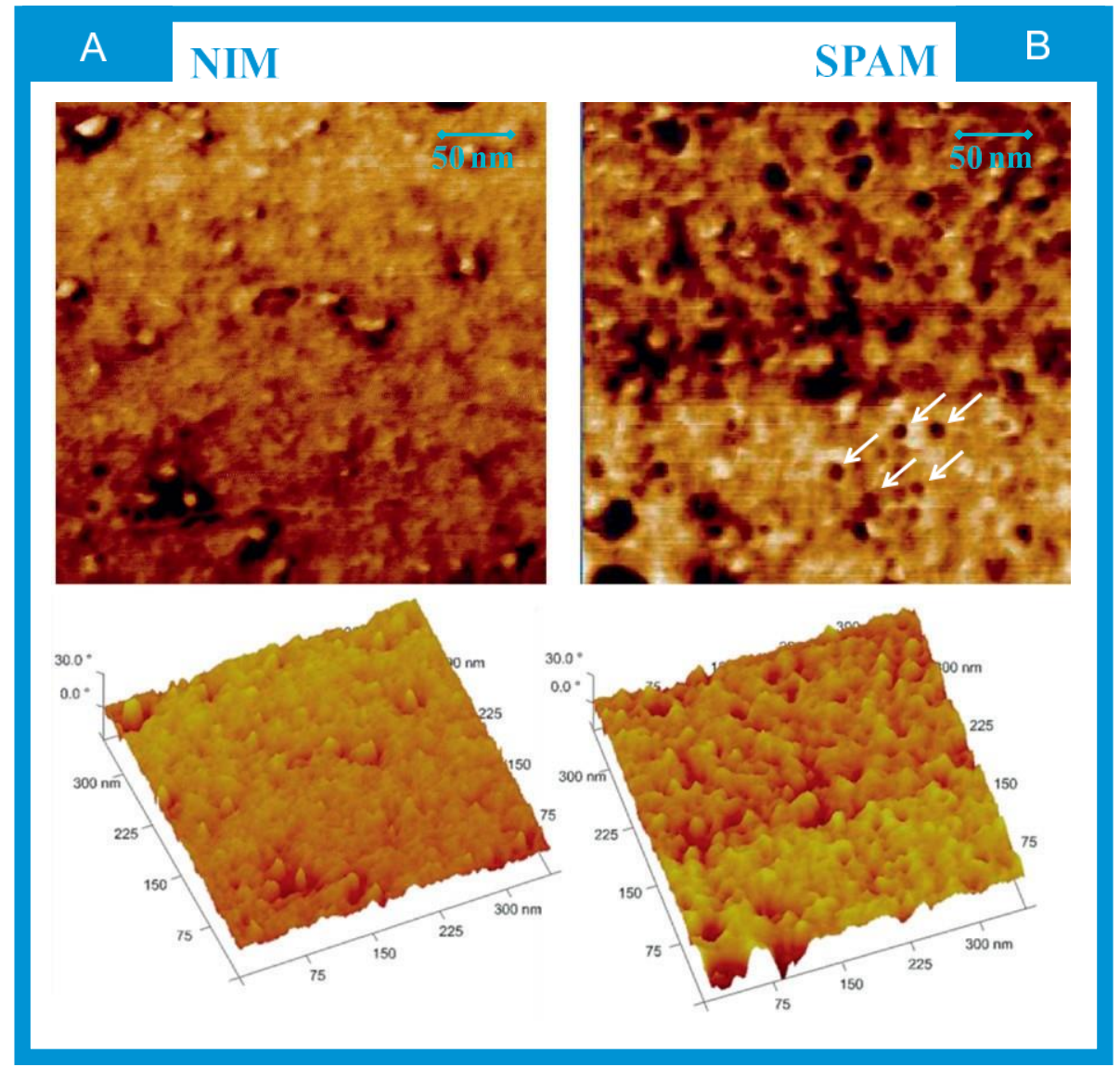

Fig. 4. AFM tapping mode images showing $300 \mathrm{~nm}$ x $300 \mathrm{~nm}$ scan after proteinase $\mathrm{K}$ treatment of (A) NIM and (B) SPAM surfaces. AFM images processed using the Nanorule program to show the 3-dimensional topography.

measurements. SPAM and NIM materials were assembled on the SPR planar gold chips following the same procedure as that described for the SPEs.

Clear differences were observed between the images of NIM and SPAM structures (Fig. 4). Only SPAM material showed cavities (these were never present in the NIM material), attributed to the plastic antibodies. About $90 \%$ of these cavities ranged between 3.5 and $10 \mathrm{~nm}$. Considering that a single Myo molecule is $4.5 \mathrm{~nm} \times 3.5 \mathrm{~nm} \times 2.5 \mathrm{~nm}$ (Lin et al., 2007), this indicated that under the imprinting conditions Myo existed both in monomeric and dimeric states. In addition, the pores of the acrylamide gels range between 21 and $200 \mathrm{~nm}$ (Stellwagen, 1998) which indicated that the AFM images obtained from tapping modes were in fact cavities in the template. A good correlation with the electrochemistry data further confirms this observation.

\subsection{Analytical performance of the sensor}

The analytical performance of Myo sensory materials was evaluated by recording calibration curves in EIS and SWV measurements (Fig. 5). Among other voltammetric methods, SWV offers the advantages of high sensitivity to surface-confined electrode reactions, along with suitable detection capabilities and rapidity. EIS readings may be straightforward for low cost and on-site analysis.

EIS calibration curves plotted the $R_{\mathrm{ct}}$ of SPAM or NIM Au-SPE sensors against logarithm Myo concentration (Fig. 5A). Increasing concentrations of Myo increased the diameter of the semicircles in the Nyquist plots (Figure S2-A), indicating that Myo bound to the sensory layer increased the charge-transfer resistance of the probe. This was as expected from the previous EIS studies, accounting for an increased $R_{\mathrm{ct}}$ when Myo was bound to the sensing material and a decreased $R_{\mathrm{ct}}$ when Myo was removed from it. The typical calibration plot of SPAM sensors shows linear behavior down to $3.5 \mathrm{mg} / \mathrm{mL}$, with an average slope of $461.4 \mathrm{O} /$ decade [Myo, $\mathrm{mg} / \mathrm{mL}$ ] and squared correlation coefficients 40.999 . The detection limit was $1.5 \mathrm{mg} / \mathrm{mL}$, corresponding to the concentration of the cross-section of the two linear parts of the response function (Buck and Lindner, 1994). The average and the relative standard deviation of the blank were 300 and $14 \mathrm{O}$, respectively. The standard deviation of repeated assays was $\mathbf{0} 5 \%$.

In SWV assays, Myo binding was revealed by a decrease in the typical anodic peak current of redox probe solutions (Figure S2-B). Higher Myo concentrations yielded smaller peaks. A linear pattern against $\log [\mathrm{Myo}]$ was observed for concentrations down to $0.58 \mathrm{mg} / \mathrm{mL}$ (Fig. 5B), a value that lies within the physiological levels expected by the first hour after cardiac damage. This linear behavior was observed up to $0.58 \mathrm{mg} / \mathrm{mL}$. The limit of detection was calculated similarly to EIS studies, and was equal to $0.28 \mathrm{mg} / \mathrm{mL}$. The average and the standard deviation of the blank were 74.0 and $3.7 \mathrm{~mA}$, respectively. The relative standard deviation of repeated assays was $\mathbf{0} \%$.

The NIM sensor displayed an inconsistent response over the range of the calibration curves, both in EIS and SWV assays (Fig. 5A and B). These results indicated that the non-specific interaction (the only kind existing in NIM surfaces) between the template and polymer did not control the electrochemical response. Only monomers polymerized in the presence of a template should have 


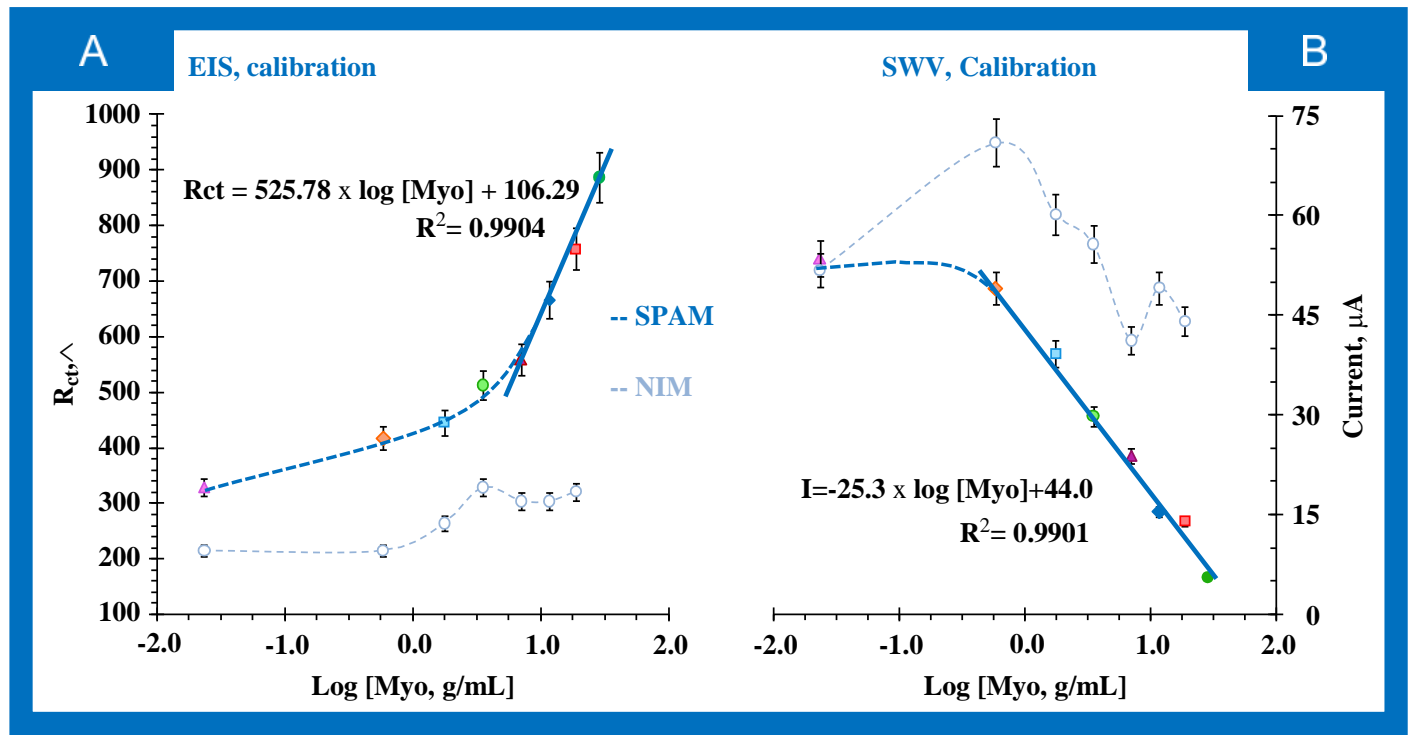

Fig. 5. Calibration curves of SPAM and NIM based Au-SPE biosensors obtained by EIS (A) and SWV (B) measurements in $5.0 \mathrm{mM}[\mathrm{Fe}(\mathrm{CN}) 6]^{3-}$ and $5.0 \mathrm{mM}[\mathrm{Fe}(\mathrm{CN}) 6]^{4-}$, in MES buffer $\mathrm{pH} 4.5$, with different concentrations of Myo (in $\mathrm{mg} / \mathrm{mL}$ ). Coefficient of variation of presented data $05 \%$.

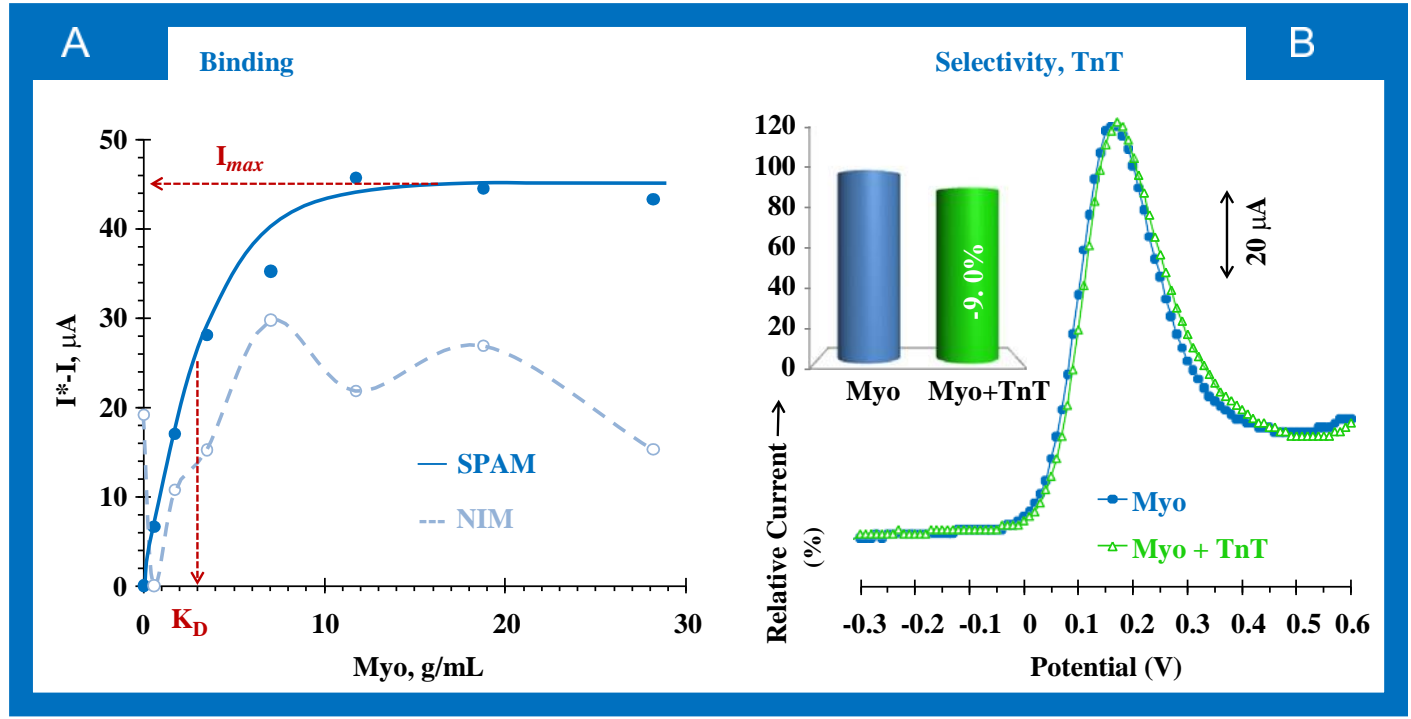

Fig. 6. Graphical representation of the Langmuir isotherm plot (A) for SPAM (with $I_{\max }$ and $K_{\mathrm{D}}$ values) and NIM materials, and the selectivity data for Tnt (B).

imprinted sites, and these sites are clearly controlling the interaction between Myo and SPAM.

\subsection{Binding isotherm}

The calibration of the SPAM biosensor in SWV follows a hyperbolic response (Fig. 6A) approaching the typical behavior of antibody/antigen interactions (Moreira et al., 2011b; Yildirim et al., 2012). The parametric data over the Langmuir equation $\left(I_{\max }\right.$ and $K_{\mathrm{D}}$ in Eq. (1)) assess the biosensor performance in terms of template rebinding. $I_{\max }$ reflects the differences in the amount of Myo bound to the sensor surface and $K_{\mathrm{D}}$ (the dissociation constant) shows the concentration of template providing half of the maximum response $\left(I_{\max }\right)$, thus measuring how well Myo complexes with the plastic antibody material and the affinity with which it occurs. If the $K_{\mathrm{D}}$ is low it indicates a large binding affinity, as the reaction will approach the maximum response more rapidly. On the contrary, a high $K_{\mathrm{D}}$ indicates that the plastic anibody does not bind as efficiently with the Myo, and only higher concentrations of Myo are able to saturate the response of the plastic antibody surface. In general, changes in $K_{\mathrm{D}}$ are sensitive to variations in protein access/binding (Sasso et al., 1990; Rothwell et al., 2010) and may be interpreted here in terms of barriers to antigen-artificial antibodyaccess.

Fitting the data to a hyperbolic function (Langmuir), where $\left(I^{*}-I\right)$ represents the binding adsorption for each concentration value of Myo and $I^{*}$ the maximum current observed in all ranges of concentration studied, gave values of $K_{\mathrm{D}} 1 / 4370.5 \mathrm{mM}$ and $I_{\max } 1 / 44472$ nA with a chi-square of 26.5 for the SPAM (Fig. 6A). The NIM binding data did not fit to the Langmuir model, since a chi-square of 556 was obtained. Overall, these results showed that the SPAM sensor displayed much higher affinity to Myo than NIM, showing a controlled/predictable response against Myo concentration. Since the differences between NIM and SPAM are only the absence/presence of Myo tailored sites on the receptor structure, respectively, the above observations pointed out that the non-specific interaction has been majorly reduced by introducing the charged monomers upon the binding sites. 


\subsection{Selectivity study}

The selectivity of the sensor is of great importance for a successful analytical application and was evaluated by SVW measurements. The interfering species tested were selected among those that may be found in biological fluids, such as TnT, BSA (serum), and urea (urine).

A competitive assay was used to test their interference in a $2.0 \times 10^{-7} \mathrm{~mol} / \mathrm{L}$ Myo solution. For this purpose, the response of the SPAM sensors was checked for solutions having only Myo or Myo pinterfering species, where a competition between Myo and interfering species for the same binding sites was established. A 5 min contact between the SPAM surface and each solution was allowed. Each assay was conducted in a different Au-SPE/SPAM sensor, in order to avoid a cross contamination from previously adsorbed Myo or interfering compound.

The average \% deviation produced by each interfering species in pure Myo solutions were $6.0 \%$ for TnT, $10.9 \%$ for BSA and $8.7 \%$ for urea. A typical voltammogram with the corresponding average $\%$ deviation for TnT may be seen in Fig. 6B, and the others are shown as supplementary material (Figure S3). The relative standard deviation of repeated assays was $\mathbf{0} 5 \%$.

\subsection{Myo assay}

The standard addition method was applied to determine Myo in spiked diluted serum samples having Myo concentrations ranging from 0.59 to $18.77 \mathrm{mg} / \mathrm{mL}$ Myo. This was performed in triplicate by SWV measurements. A good agreement was found between added and found amounts of Myo, with recoveries lying within $94 \%$ and $97 \%$ respectively. The relative standard deviation obtained for reproduced assays was $\mathbf{0} 5.8 \%$ and for repeated assays $\mathbf{0} 5 \%$, thus confirming the good precision of this new analytical approach. These results show great expectations around this SPAM network, also acting as a promising tool for direct applications in point-of-care context.

\section{Conclusions}

Compared to previous Myo plastic antibodies described in the literature (Moreira et al., 2011b; Wang et al., 2008), this is the first time an imprinted material is able to recognize the target protein mostly by its specific binding sites. As far as we are aware this is the first time that a non-imprinted polymer showed an absence of dose response behavior with a target molecule protein, consistent with the non-specific binding to the polymer being minimal. Furthermore, we believe that it is the first time that the imprinted sites are seen in the AFM images, showing cavities of a comparable size to the imprinted protein or only small aggregates.

Overall, it seems that the SPAM approach proposed here concerning the use of charged-monomers offers a promising alternative to the design of new surface confined plastic antibodies for proteins. Although the limit of detection provides proof of concept for using the device in point-of-care context, further developments will be necessary for practical application in a clinical setting.

\section{Acknowledgments}

One of the authors (FTCM) gratefully acknowledges Fundaeao para a Ciência e Tecnologia (FCT) for the financial support (PhD grant reference SFRH/BD/66735/2009, entitled "Fast screening for early diagnostic of heart ischemic episodes").

\section{Appendix A. Supporting information}

Supplementary data associated with this article can be found in the online version at http://dx.doi.org/10.1016/j.bios.2013.02. 012 .

\section{References}

Andersson, L.I., 2000. Journal of Chromatography B 739 (1), 163-173.

Bonini, F., Piletsky, S., Turner, A.P.F., Speghini, A., Bossi, A., 2007. Biosensors and Bioelectronics 22 (9-10), 2322-2328.

Borrebaeck, C.A.K., 2000. Immunology Today 21 (8), 379-382.

Bossi, A., Bonini, F., Turner, A.P.F., Piletsky, S.A., 2007. Biosensors and Bioelectronics 22 (6), 1131-1137.

Bossi, A.M., Sharma, P.S., Montana, L., Zoccatelli, G., Laub, O., Levi, R., 2012. Analytical Chemistry 84 (9), 4036-4041.

Brunori, M., Bourgeois, D., Vallone, B., 2004. Journal of Structural Biology 147 (3), 223-234.

Buck, R.P., Lindner, E., 1994. Pure and Applied Chemistry 66 (12), 2527-2536.

Busi, E., Basosi, R., Ponticelli, F., Olivucci, M., 2004. Journal of Molecular Catalysis A: Chemical $217(1-2), 31-36$.

Chen, L., Xu, S., Li, J., 2011. Chemical Society Reviews 40 (5), 2922-2942.

Hillberg, A.L., Tabrizian, M., 2008. IRBM 29 (2-3), 89-104.

Jiang, K.Y., Schadler, L.S., Siegel, R.W., Zhang, X.J., Zhang, H.F., Terrones, M., 2004 Journal of Materials Chemistry 14 (1), 37-39.

Katz, A., Davis, M.E., 2000. Nature 403 (6767), 286-289.

Kriz, D., Ramstrom, O., Mosbach, K., 1997. Analytical Chemistry 69 (11), A345A349.

Lass-Napiorkowska, A., Heyduk, E., Tian, L., Heyduk, T., 2012. Analytical Chemistry 84 (7), 3382-3389.

Liao, P.-C., Tyan, Y.-C., Wang, C.-Y., Hsu, J.-F., Chou, T.-C., Lin, H.-Y., 2009. Journal of Biomedical Materials Research Part A 91A (2), 597-604.

Lin, H.-Y., Rick, J., Chou, T.-C., 2007. Biosensors and Bioelectronics 22 (12), 32933301.

Lu, C.-H., Zhang, Y., Tang, S.-F., Fang, Z.-B., Yang, H.-H., Chen, X., Chen, G.-N., 2012. Biosensors and Bioelectronics 28 (1), 243-250.

Moreira, F.T.C., Dutra, R.A.F., Noronha, J.P.C., Cunha, A.L., Sales, M.G.F., 2011 a. Biosensors and Bioelectronics 28 (1), 243-250.

Moreira, F.T.C., Dutra, R.A.F., Noronha, J.P.C., Sales, M.G.F., 2011b. Biosensors and Bioelectronics 26 (12), 4760-4766.

Panagopoulou, M.A., Stergiou, D.V., Roussis, I.G., Prodromidis, M.I., 2010. Analytica Chemistry 82 (20), 8629-8636.

Piletsky, S.A., Piletska, E.V., Chen, B.N., Karim, K., Weston, D., Barrett, G., Lowe, P., Turner, A.P.F., 2000. Analytical Chemistry 72 (18), 4381-4385.

Reddy, S.M., Sette, G., Phan, Q., 2011. Electrochimica Acta 56 (25), 9203-9208.

Rimmer, S., 1998. Chromatographia 47 (7-8), 470-474.

Rothwell, S.A., Killoran, S.J., O'Neill, R.D., 2010. Sensors 10 (7), 6439-6462.

Ruigrok, V.J.B., Levisson, M., Eppink, M.H.M., Smidt, H., van der Oost, J., 2011. Biochemical Journal 436, 1-13.

Sasso, S.V., Pierce, R.J., Walla, R., Yacynych, A.M., 1990. Analytical Chemistry 62 (11), 1111-1117.

Savina, I.N., Dainiak, M., Jungvid, H., Mikhalovsky, S.V., Galaev, I.Y., 2009. Journal of Biomaterials Science, Polymer Edition 20 (12), 1781-1795.

Shi, H.Q., Tsai, W.B., Garrison, M.D., Ferrari, S., Ratner, B.D., 1999. Nature 398 (6728), 593-597.

Shiomi, T., Matsui, M., Mizukami, F., Sakaguchi, K., 2005. Biomaterials 26 (27), 5564-5571.

Stellwagen, N.C., 1998. Electrophoresis 19 (10), 1542-1547.

Takeuchi, T., Hishiya, T., 2008. Organic and Biomolecular Chemistry 6 (14), 2459-2467.

Turner, N.W., Jeans, C.W., Brain, K.R., Allender, C.J., Hlady, V., Britt, D.W., 2006. Biotechnology Progress 22 (6), 474-1489.

Wang, Y., Zhou, Y., Sokolov, J., Rigas, B., Levon, K., Rafailovich, M., 2008. Biosensors and Bioelectronics 24 (1), 162-166.

Whitcombe, M.J., Chianella, I., Larcombe, L., Piletsky, S.A., Noble, J., Porter, R., Horgan, A., 2011. Chemical Society Reviews 40 (3), 1547-1571.

Ye, L., Mosbach, K., 2001. Reactive and Functional Polymers 48 (1-3), 149-157.

Yildirim, E., Turan, E., Caykara, T., 2012. Journal of Materials Chemistry 22 (2), 636-642.

Zayats, M., Kanwar, M., Ostermeier, M., Searson, P.C., 2011. Macromolecules 44 (10), 3966-3972.

Zdyrko, B., Hoy, O., Luzinov, I., 2009. Biointerphases 4 (2), FA17-FA21. 